\title{
Effects of steeping variables and substrate mesh size on starch yield extracted from oil palm trunk
}

\begin{abstract}
Large area of oil palm plantation in Malaysia generated significant amount of oil palm biomass such as oil palm trunk. This material is assumed to possess multipurpose utilization, including a starch-producing raw material. This work is aimed to determine the total extractable starch from oil palm trunk (OPT) using commercial steeping methods. The extraction methods used in this study include steeping the OPT in sodium metabisulphite (Na2S2O5) alone and with the addition of lactic acid (C3H6O3). The effect of process parameters: incubation temperature (IT) (room temperature at $26{ }^{\circ} \mathrm{C}, 40{ }^{\circ} \mathrm{C}, 50{ }^{\circ} \mathrm{C}$ ), incubation period (IP) (24 h, 36 h, 48 h) and substrate mesh size (MS) (20 mesh, 40 mesh, 60 mesh) were evaluated for their effect on starch yield. The experimental results indicated that with the addition of lactic acid during steeping improved the starch yield $(3.61 \%)$ as compared to that of steeping using sodium metabisulphite alone $(0.92 \%)$. The effect of substrate mesh size is more significant than process parameters: incubation temperature and incubation period on the starch yield. The contour plot was derived from the equation and applied to determine the interactive effects of IT $\times$ IP, IT $\times$ MS and IP $\times$ MS. Among the interactions, IT $\times$ MS is more significant than IT $\times$ IP, and IP $\times$ MS on the starch yield. A second-order regression equation was developed for the response as a function of independent coded parameters $(\mathrm{R} 2=0.91)$. Conclusively, starch recovery increased with increase of OPT mesh size and steeping incubation temperature.
\end{abstract}

Keyword: Oil palm trunk; Starch yield; Incubation temperature; Incubation period; Mesh size 